\title{
Switching Schemas: Do Effects of Mindless Interactions With Agents Carry Over to Humans and Vice Versa?
}

\author{
John A. Velez ${ }^{1}$, Travis Loof ${ }^{2}$, Casey A. Smith ${ }^{3}$, Joshua M. Jordan ${ }^{4}$, \\ Jonathan A. Villarreal ${ }^{3}$, \& David R. Ewoldsen ${ }^{5}$ \\ 1 Communication Science Unit, School of Media, Indiana University, Bloomington, IN 47405, USA \\ 2 Media \& Journalism, College of Arts and Sciences, University of South Dakota, Vermillion, SD 57069, USA \\ 3 College of Media \& Communication, Texas Tech University, Lubbock, TX 79409, USA \\ 4 Media Arts \& Sciences, School of Media, Indiana University at Bloomington, Bloomington, IN 47405, USA \\ 5 Media \& Information, College of Communication Arts and Sciences, Michigan State University, East Lansing, Ml 48824, \\ USA
}

\begin{abstract}
The Revised Ethopoeia Concept proposes that agents should activate human schema in the form of social expectations and rules. However, studies have demonstrated inconsistent reactions to agents and avatars, potentially due to the activation of a nonhuman schema that constrains people's ability to mentally connect with agents. We first examined whether the schema activated during agent or avatar game play were consistent with and influenced a subsequent interaction with a human. Results suggested that schemas activated across consecutive interactions with an agent and then a human were incongruent, while avatars successfully influenced subsequent interactions with humans (i.e., prosocial behaviors) by evoking congruent schemas. A second experiment corroborated this by demonstrating a match between schemas activated during game play with agents and subsequent interactions with another human-like but nonhuman entity (i.e., artificial intelligence). Additional results and implications related to switching schemas in consecutive interactions with agents and humans are discussed.
\end{abstract}

Keywords: Avatars and Agents, Bounded Generalized Reciprocity, Computers as Social Actors, Prosocial Behaviors, Social Game Play, Computer Games

doi:10.1093/jcmc/zmz016

Social interactions with agents (i.e., computer-controlled digital representations; Nowak \& Fox, 2018) are becoming ubiquitous. Programmers are making agent software more sophisticated, enabling

Corresponding author: John A. Velez; e-mail: johnvele@iu.com

Editorial Record: First manuscript received on 1 November 2018; Revisions received on 3 March 2019 and 23 May 2019; Accepted by Prof. Nicole Kraemer on 19 July 2019; Final manuscript received on 26 July 2019 
them to impersonate specific human characteristics with increasing accuracy. A body of research suggests that agents are capable of eliciting social responses from interactants, such as increased liking of a technology's assigned persona, abiding by social norms while in conversation, and increasing selfdisclosure (i.e., the Media Equation; Reeves \& Nass, 1996; see Nowak \& Fox, 2018). However, as the applications of agents proliferate, an increasingly important question is how these social responses to agents will influence people's subsequent interactions with humans (i.e., consecutive interactions). Indeed, communication is a dynamic process, and research and theory need to reflect the dynamic nature of communication across time (Lang \& Ewoldsen, 2010).

As predicted by the theory of Bounded Generalized Reciprocity (BGR; Yamagishi, Jin, \& Kiyonari, 1999), the social strategies video game players use with other avatars (i.e., human-controlled digital representations) can guide subsequent prosocial behaviors in non-mediated interactions. Video games are also perhaps the most common source of interaction with agents that are, oftentimes, indistinguishable from other avatars. If people have human, social responses to agents, then it is possible that nonhuman entities can shape the social strategies people use to guide their prosocial behaviors in subsequent human interactions as well. However, research also suggests that interactions with agents may differ from those with avatars on crucial elements that may disrupt the connection between game play and subsequent effects on prosocial behaviors. Research suggests that people struggle to connect with agents mentally (see Fox et al., 2015), potentially because people use different mental schemas for agents, compared to avatars (Lim \& Reeves, 2010; Merritt, 2012). Although we may initially have social responses to agents, the utilization of different schema for agents, compared to avatars, may change our subsequent social implications for interacting with humans.

We used two studies to compare the inter-group processes (e.g., in-group favoritism, which is a naturally occurring bias that favors one's own group, compared to other groups; Tajfel, Bilig, Bundy, and Flament, 1971) that occur during agent and avatar interactions in the context of video game play and how such processes can influence subsequent prosocial behaviors. The first study examined the social strategies people use in prosocial interactions with a stranger that occur after playing either cooperatively or competitively, with either an agent or an avatar. A follow-up study further explored social strategies and prosocial behaviors by providing participants with a subsequent partner (i.e., a stranger or a different agent) that either matched or mismatched the schema used during game play with an agent or avatar.

\section{Experiment 1}

The phenomenon known as in-group favoritism is the purported mechanism behind cooperative game play's effect on subsequent behavior. The theory of BGR (Yamagishi et al., 1999) suggests that reciprocity expectations shaped during social game play will predict players' post-game prosocial behaviors towards teammates (Velez, 2015) and even towards strangers with whom they did not interact (e.g., Greitemeyer \& Cox, 2013; Greitemeyer, Traut-Mattausch, \& Osswald, 2012). According to the theory of BGR, the formation of teams in social game play will activate the Group Heuristic. The Group Heuristic is a purported cognitive heuristic that leads people to automatically believe that in-group members will reciprocate favors and other positive behaviors (i.e., high reciprocity expectations), particularly compared to out-group members. Importantly, these expectations of reciprocity generalize to members of the group, and not simply the individual who engaged in reciprocal behavior. The theory states that those who violate the Group Heuristic (i.e., do not initially engage in positive behaviors or reciprocate positive behaviors) lose the opportunity to receive favors in future in-group interactions (for more, see Velez, 2018). For example, research has found that social game play can signify people's trustworthiness 
in subsequent, non-video game interactions and that teammates who violate reciprocity expectations receive fewer post-game prosocial behaviors than teammates who confirm expectations (Velez, 2015).

\section{Cooperative and competitive game play}

As discussed above, research suggests that the benefits of cooperative play are mediated by increased reciprocity expectations, as predicted by BGR. Research has found that cooperating, compared to competing, leads to increased reciprocity expectations of teammates (Waddell \& Peng, 2014), prosocial outcomes (Ewoldsen et al., 2012; Velez, Mahood, Ewoldsen, \& Moyer-Gusé, 2014), and decreased aggressive behaviors (Study 1 in Velez, Greitemeyer, Whitaker, Ewoldsen, \& Bushman, 2016), although other studies have found inconsistent effects (Schmierbach, 2010; Velez et al., 2016). Research also suggests that reciprocity expectations formed in one interaction can also influence expectations of a subsequent stranger (i.e., not a teammate; Bettenhausen \& Murnighan, 1991). Although not explicitly stated in BGR, the transference of expectations from one interaction to subsequent interactions with a stranger is consonant with the theory's predictions. Indeed, cooperative game play, compared to solo game play, has led to higher reciprocity expectations and prosocial behaviors towards strangers (Greitemeyer \& Cox, 2013; Greitemeyer, Traut-Mattausch, \& Osswald, 2012). Although the influence of cooperative game play on reciprocity expectations of strangers has not been specifically compared to that of competitive game play, the above research suggests that expectations should mediate differences in prosocial behaviors towards strangers.

H1: Participants who played a video game cooperatively with an avatar will have higher reciprocity expectations of a stranger, compared to participants who (a) played competitively against an avatar or (b) did not play a game at all.

H2: Participants who played a video game cooperatively with an avatar will behave more prosocial towards a stranger, compared to participants who (a) played competitively against an avatar or (b) did not play a game at all.

H3: Participants' reciprocity expectations of a stranger will mediate the differences in prosocial behaviors exhibited after cooperative game play with an avatar, compared to participants who (a) played competitively against an avatar or (b) did not play a game at all.

\section{Social game play and computers as social actors}

BGR provides a theoretical framework for understanding how interactions between players (i.e., avatars) influence subsequent prosocial behaviors. A theoretical framework for consecutive human-human interactions (i.e., two or more interactions between the same or different pairs of humans), such as BGR, can be used as a foundation for examining the influence of human-agent interactions on future behaviors (see Krämer, Eimler, von der Pütten, \& Payr, 2011). Indeed, a hallmark of the Computers as Social Actors (CASA) paradigm is to take "findings and experimental methods from the social sciences" and apply them to human-media interactions, with the prediction that the results will be the same (Reeves \& Nass, 1996, p. 28). Numerous studies have supported the basic premise of CASA by eliciting common social principles from people interacting with computers, such as reciprocity, stereotypes, flattery, politeness, praise, and criticism (for a review, see Krämer, Rosenthal-von der Pütten, \& Hoffmann, 2015). Research suggests the social reactions to computers with simple social cues are more than anthropomorphism, but rather a mindless process, termed "ethopoeia," in which people automatically apply social expectations and rules to any human-like entity without considering the appropriateness of their actions (Nass \& Moon, 2000). 
Agents can offer a complex and rich array of human characteristics, including a digital human representation that exhibits complicated verbal and non-verbal behaviors, particularly in comparison to the computers in early CASA research (see Bergmann, Branigan, \& Kopp, 2015). As such, a revised version of the ethopoeia concept was introduced that can account for the continuum of social behaviors agents offer. The Revised Ethopoeia Concept acknowledges that automatic and unconscious processing will be stronger when an entity displays more social cues (Nass \& Yen, 2010). Indeed, research has demonstrated that people experience richer social interactions with agents (e.g., longer verbal responses and greater perceptions of mutual awareness) and avatars when the other party exhibits more social cues (Appel, von der Pütten, Krämer, \& Gratch, 2012), such as responsive non-verbal behaviors (von der Pütten, Krämer, Gratch, \& Kang, 2010) and emotional expressions (de Melo, Gratch, \& Carnevale, 2013).

When it comes to consecutive social interactions, research has demonstrated that human--agent interactions influence peoples' subsequent behaviors towards the same agent, in accordance with the theory of BGR. For example, agents' behaviors in social dilemma games have been shown to evoke reciprocal processes in humans for future interactions with the same agent (Nass, Fogg, \& Moon, 1996; Parise, Kiesler, Sproull, \& Waters, 1999; Straßmann, von der Pütten, \& Krämer, 2018). Although consecutive interactions with agents (human-agent to human-agent) conform to the predictions of BGR, it is unknown whether interactions with agents lead to the same results when the target of subsequent behaviors is a human (human-agent to human-human). If BGR processes can occur with agents and the Revised Ethopoeia Concept is correct, then the social implications of an agent and avatar with the same social cues (i.e., behavioral realism) should be similar, if not identical, for subsequent prosocial behaviors towards a human. However, research has demonstrated that people's social reactions to avatars and agents are not always congruent (see Fox et al., 2015). Specifically, research has shown that agents fail to elicit the same emotions as avatars (de Melo et al., 2013). People are less likely to feel guilty about cheating an agent (de Melo, Marsella, \& Gratch, 2016) or show emotional distress and empathic concern towards a robot being mistreated (von der Pütten et al., 2014). Likewise, people report playing video games with an avatar as more physiologically arousing (Lim \& Reeves, 2010), enjoyable, and evoking more feelings of presence than playing with an agent (Guadagno, Blascovich, Bailenson, \& McCall, 2007; Weibel, Wissmath, Habegger, Steiner, \& Groner, 2008).

Researchers have proposed that agents do not activate metalizing processes that are critical and ubiquitous to human-human interactions. That is, people do not automatically feel the need to ascribe independent mental states to agents, who are incapable of "thoughts, beliefs, desires and intentions, different from our own" (Lim \& Reeves, 2010, p. 58). While research has demonstrated that interactions with avatars can activate the brain regions associated with mentalizing, interactions with agents do not produce the same brain patterns (Gallagher, Jack, Roepstorff, \& Frith, 2002; Kircher et al., 2009). This consistent finding has been interpreted as evidence that participants were more heavily engaged in cognitive tasks to better understand and predict the avatar's behaviors (i.e., mentalizing), as compared to the agent's behaviors (e.g., Lim \& Reeves, 2010).

The lack of mentalizing for agents may be the result of people using different schemas for avatars and agents (Merritt, 2012). When playing with a presumed avatar, players may assign more mind to their partner (e.g., Waytz, Gray, Epley, \& Wegner, 2010) and naturally apply a human schema to the interaction that requires and facilitates mentalizing processes. However, when playing with a presumed agent, players may use a different schema, perceived to be more appropriate for interactions with nonhuman agents, that does not require or facilitate mentalizing (Lim \& Reeves, 2010; Merritt, 2012). For example, research has shown that people do not categorize avatars and agents into the same group and that intergroup biases can emerge based on the perceived agency of digital representations (see de Melo, Carnevale, Gratch, 2014). 
Applying a nonhuman schema to agents and the resulting lack of mentalizing may be pivotal for consecutive social interactions. People use past experiences, but use recent interactions in particular (e.g., Bettenhausen \& Murnighan, 1991), to inform their reciprocity expectations and behaviors towards another person. However, if agents are not grouped with other humans (i.e., avatars; de Melo et al., 2014), then perhaps people will not find prior interactions with agents applicable or informative for guiding their prosocial behaviors towards a human. Yet, it is unknown whether the perceived agency of a video game character will change how previous interactions affect future prosocial behaviors or whether the identical behavioral realism of an avatar and agent will lead to similar results, as predicted by the Revised Ethopoeia Concept. Therefore, the following research questions were posed. First, the ability of agents to evoke reciprocal processes that are prosocial for subsequent strangers was examined (RQ1). Secondly, agents and avatars were directly compared by examining participants' general reciprocity expectations (i.e., expectations that a stranger will reciprocate any behavior, regardless of the valence; RQ2). General reciprocity expectations are indicative of agents' and avatars' potential to evoke any type of reciprocal process beyond specifically reciprocating prosocial behaviors. Finally, the differences in reciprocity expectations and prosocial behaviors after game play with agents and avatars were compared (RQ3).

RQ1: Will participants who play a video game cooperatively with an agent behave more prosocially towards a stranger, mediated by higher reciprocity expectations, compared to participants who (a) play competitively against an agent or (b) do not play a game at all?

RQ2: Will social video game play with agents change subsequent general reciprocity expectations of a stranger, compared to social game play with avatars?

RQ3: Will social game play with agents have different effects on participants' (a) reciprocity expectations and (b) consequent prosocial behaviors towards a stranger, compared to social game play with avatars?

\section{Methods}

\section{Participants}

Participants were 184 students $\left(57.9 \%\right.$ females; $\left.M_{\mathrm{age}}=21.2, S D=2.06\right)$ recruited from a large Southwestern University who received course credit for their participation (see Table 1 for the number of participants per condition). Four participants were removed because their scores on the main dependent variable (i.e., prosocial behavior) were identified as outliers via boxplots. ${ }^{1}$

\section{Procedure}

This study employed a 2 (agency: avatar vs agent) x 2 (game play: cooperative vs. competitive) experiment, with an additional control condition in which no game play occurred. Participants watched a short tutorial video before playing the video game for 15 minutes, then completed a post-test survey and engaged in a modified prisoner's dilemma task with a stranger.

\section{Stimuli}

The video game, Team Fortress 2, is a multiplayer, first-person shooter game for a personal computer. A small, farm-themed map, named Harvest, was used. The game mode was King of the Hill, in which 
Table 1 Participants in Each Experiment Across Conditions

\begin{tabular}{ll}
\hline & Participants \\
\hline Experiment 1 & \\
Cooperative avatar & 36 \\
Cooperative agent & 42 \\
Competitive avatar & 33 \\
Competitive agent & 33 \\
Control & 40 \\
Experiment 2 & \\
Avatar/human & 25 \\
Avatar/AI & 21 \\
Agent/human & 27 \\
Agent/AI & 33 \\
\hline
\end{tabular}

Note: $\mathrm{AI}=$ artificial intelligence.

players battle to control a specific area of the map. Once the area was held for a period of time, the game started a new round, until 15 minutes were completed.

\section{Manipulations}

\section{Agency}

Participants played with or against a trained confederate in all conditions. Participants in the agent conditions were told their partner was a "computer-controlled character" but, in reality, they were playing with a confederate who was sitting in the next room. Participants in the real-player character conditions were seated in their cubicle before their confederate entered the lab, was seated in another cubicle, and was given instructions identical to those given to the participant. Although participants could hear the confederate enter and receive instructions, they were not able to see the confederate at any time. This was to ensure the gender and appearance of the confederate did not influence participants' perceptions of their teammate.

\section{Game play}

In the cooperative conditions, participants played as the "heavy" character, which is a large man with a minigun. The main purpose of this character is to deal and receive large amounts of damage. The participants' teammate was the "medic" character, which is a doctor with a "Syringe Gun" that shoots syringes for damage and a "Medi Gun" that shoots a ray capable of healing a teammate. Confederates were instructed to heal participants at all times and to do so within participants' line of sight. Previous research suggests that cooperative contexts are not sufficient to elicit post-game prosocial behaviors, and that teammates must perform helpful behaviors during game play (Velez, 2015). Participants cooperated with their partner (i.e., an avatar or agent) against another team of two agents, consisting of the same duo of characters (i.e., the heavy and medic). Participants were informed the other team consisted of computer-controlled characters. In the competitive conditions, the participant and confederate both played the heavy character against each other. 


\section{Measures}

General reciprocity expectations

Participants' general reciprocity expectations were measured using one item, created for the purpose of this study. Participants' expectations that their interaction partner would reciprocate any behavior, regardless of valence, was measured by their agreement with the statement "I expect my transaction partner to donate a similar amount of money as I donate to her/him" (measured on a scale from 1 [strongly disagree] to 7 [strongly agree]).

\section{Prosocial behaviors}

Participants' prosocial behavior was measured using a modified prisoner's dilemma game (Ewoldsen et al., 2012; Van Lange \& Kuhlman, 1994). Participants were told they would be engaging in a money transaction with a stranger. Participants were told they had 10 dimes and they could keep or donate any number of dimes to their transaction partner. Additionally, donated dimes would double in value for their transaction partner, but any dimes kept would not. Participants were told their transaction partner was making the same donation decision at the same time, before indicating via an online questionnaire how many dimes they would like to donate.

\section{Reciprocity expectations}

Participants' prosocial reciprocity expectations were measured using 1 item, pertaining to participants' expectations from their interaction partners in the prisoner's dilemma game (Greitemeyer \& Cox, 2013; Greitemeyer et al., 2012; Rothmund, Gollwitzer, \& Klimmt, 2011; Velez, 2015). Participants were asked "out of the 10 dimes possible to donate, how many dimes do you think your transaction partner (again, not a person in this room) will choose to donate to you?"

\section{Results}

\section{Manipulation check}

At the end of the study, participants were asked about their teammate ("for today's video game session, who was on your team?") or opponent ("who were you playing against?"). Participants chose between "a computer-controlled character or "another person." Participants who selected the incorrect option were removed from the analyses (avatar conditions $=16$; agent conditions $=5$ ) ${ }^{2}$

\section{Analyses}

Four directional (i.e., one-tailed) planned contrasts were conducted for reciprocity expectations, and then prosocial behaviors, to examine differences between cooperative game play and competitive or no game play for the avatar conditions $(\mathrm{H} 1 \mathrm{a} / \mathrm{b}$ and $\mathrm{H} 2 \mathrm{a} / \mathrm{b})$. Bonferroni adjustments were applied to each set of four contrasts (i.e., one set for reciprocity expectations and another for prosocial behaviors). Adjusted $p$ values were calculated by first dividing values by two (i.e., to account for the directionality of comparisons) and then multiplying the resulting $p$ values by 4 (i.e., the number of contrasts) to account for Bonferroni corrections. The comparisons between the agent and control conditions (RQ1a/b) were treated as two-tailed analyses and, thus, the $p$ values were only multiplied by the number of contrasts. Research questions comparing avatar and agent social game play on resulting general reciprocity expectations (RQ2), reciprocity expectations (RQ3a), and prosocial behaviors (RQ3b) were examined with two-way analyses of variance (ANOVAs), with agency and game play type entered as fixed factors. Simple main effects were examined for each two-way ANOVA using Bonferroni adjustments. 
The Statistical Package for the Social Sciences (SPSS) macro PROCESS (Hayes, 2013) was used to examine the simple mediation (Model 4) hypothesis (H3) and research question (RQ1) with 10,000 bootstrap samples. Two dummy variables that represented the planned contrasts between competitive and cooperative play (i.e., $\mathrm{d} 1$ ) and between no game play and cooperative play (i.e., d2) were created. This coding scheme represented the cooperative game play condition with a 0 (i.e., the reference category) while the competitive and no game play conditions were each represented with a 1 .

\section{Avatars: $\mathrm{H1}-\mathrm{H} 3$}

The first hypothesis predicted that playing cooperatively with avatars would lead to higher reciprocity expectations of a stranger, compared to (a) playing competitively or (b) not playing at all. Levene's test of homogeneity of variances was not significant for reciprocity expectations $(F[4,179]=.68$; $p=.606)$. Directional $t$-tests with Bonferroni adjustments indicated that playing cooperatively with an avatar $(M=7.64, S D=2.78)$ did not lead to higher reciprocity expectations than playing competitively against an avatar $(M=6.33, S D=3.01)$, but cooperative play did lead to higher reciprocity expectations when compared to not playing at all $(M=5.73, S D=3.43 ; t[179]=2.63 ; p=.018 ; d=.61)$. The first hypothesis was partially supported.

The second hypothesis predicted that playing cooperatively with avatars would result in more prosocial behavior towards a stranger, compared to (a) playing competitively or (b) not playing at all. The Levine's test of homogeneity of variances was significant for prosocial behavior $([4,179]=2.98$; $p=.021)$. Directional $t$-tests with Bonferroni adjustments indicated that playing cooperatively $(M=8.61, S D=2.25)$ only resulted in a marginal increase in prosocial behavior, compared to playing competitively $(M=7.09, S D=3.21 ; t[56.78]=2.26 ; p=.056 ; d=.55)$, but a significant increase compared to not playing at all $(M=6.28, S D=3.17 ; t[70.27]=3.73 ; p<.001 ; d=.85)$. Therefore, the second hypothesis was partially supported.

The third hypothesis predicted that the effect of cooperative play with avatars on prosocial behavior towards a stranger, compared to competitive or no game play, would be mediated by increased reciprocity expectations. Results indicated that playing cooperatively influenced participants' prosocial behavior by increasing their reciprocity expectations of strangers, compared to no game play (point estimate $=-1.32, S E=.51$; confidence interval -2.39 to -.37 ). The mediation analysis did not indicate a significant difference between cooperative and competitive game play. Therefore, the third hypothesis was partially supported.

\section{Agents: RQ1}

The first research question examined whether playing cooperatively with agents would lead to more prosocial behaviors, mediated by higher reciprocity expectations, compared to (a) playing competitively against agents or (b) not playing at all. In regard to reciprocity expectations, two-tailed $t$-tests with Bonferroni adjustments indicated no significant differences between the relevant conditions. In regard to prosocial behaviors, two-tailed $t$-tests with Bonferroni adjustments indicated that playing cooperatively with an agent led to greater prosocial behaviors $(M=8.10, S D=2.45)$, compared to not playing at all $(M=6.28, S D=3.17 ; t[73.36]=2.90 ; p=.024 ; d=.64)$, but not compared to playing competitively against an agent $(M=8.06, S D=2.72)$. Regarding the mediating role of reciprocity expectations on prosocial behaviors, mediation analyses using the same procedure for the third hypothesis did not indicate a significant, indirect effect for either dummy variable (i.e., $\mathrm{d} 1$ and $\mathrm{d} 2$ ), thus answering the first research question. 


\section{Avatars versus agents: RQ2 and RQ3}

The second research question asked whether game play with agents could change general reciprocity expectations of strangers in subsequent interactions, similar to game play with avatars. Results indicated a significant main effect for player type, such that game play with avatars led to higher general reciprocity expectations $(M=3.93, S D=1.40)$ than game play with agents $(M=3.37, S D=1.83 ; F[1,140]=4.10$; $\left.p=.045 ; \eta_{\mathrm{p}}{ }^{2}=.03\right)$. There were no other significant results.

The third research question asked whether participants' (a) reciprocity expectations and (b) prosocial behaviors towards strangers differed depending on agency and game play type (i.e., a two-way interaction). Results did not indicate any significant main or interaction effects for participants' reciprocity expectations, thus answering the first part of the third research question. Results only indicated a marginal main effect of game play type $\left(F[1,140]=3.06 ; p=.083 ; \eta_{\mathrm{p}}{ }^{2}=.02\right)$ and a marginal interaction effect between agency and game play type $\left(F[1,140]=2.79 ; p=.097 ; \eta_{\mathrm{p}}{ }^{2}=.02\right)$ for participants' prosocial behaviors. Therefore, the third research question was answered.

\section{Discussion}

The first experiment examined whether similar social processes occurred for agents and avatars and whether such processes were applied to subsequent interactions with a stranger. The interactions between avatars followed previously demonstrated social processes in video game play. Specifically, cooperative play compared to no game play confirmed participants' expectations that an avatar teammate would reciprocate prosocial behaviors and, thus, led to higher reciprocity expectations in a subsequent interaction with a stranger. These reciprocity expectations translated into more prosocial behaviors, as predicted by BGR (Yamagishi et al., 1999). Although previous research found that competitive game play reduced reciprocity expectations and prosocial behaviors between teammates, compared to cooperative play (Waddell \& Peng, 2014), participants' expectations and behaviors towards strangers were not similarly influenced in the current study. The influence of competitive game play, in general, has been shown to be weak or inconsistent when compared to cooperative play (Schmierbach, 2010; Velez et al., 2016; see comparisons across multiple Prisoner's Dilemma Game (PDG) trials in Velez et al., 2014). This may be due to players not attributing overly hostile intentions to their opponents, who are simply following the rules of the game (i.e., the competitive structure of the game mode; see Reeder, Kumar, Hesson-McInnis, \& Trafimow, 2002; Velez et al., 2016), and thereby reducing the impact of competitive play on subsequent reciprocity expectations, particularly towards a non-game partner.

The Revised Ethopoeia Concept (Nass \& Yen, 2010) suggests that BGR's predictions for consecutive social interactions should be equally applicable to agents and avatars. That is, the Revised Ethopoeia Concept predicts that identical agents and avatars should activate the same schema and, subsequently, lead to similar, if not identical, effects on reciprocity expectations and prosocial behaviors towards a stranger. Recall that in this experiment, the avatar's and agent's behaviors were both controlled by a human, which should result in highly similar behaviors by the two. Based on the Revised Ethopoeia Concept, this situation should be highly likely to activate the same schemas. However, other research has suggested people may use a nonhuman schema for agents (e.g., Merritt, 2012), which may disrupt the connection between game play and subsequent effects on prosocial behaviors towards a stranger. Therefore, the pattern of results between the agent and avatar conditions were compared.

Results demonstrated several discrepancies between reciprocity expectations and prosocial behaviors towards strangers after game play with agents versus avatars (see Figure 1). Playing against an agent had a relatively small effect on participants' general reciprocity expectations of strangers, regardless of cooperative or competitive play, suggesting the nuances of interactions with agents are not being applied 


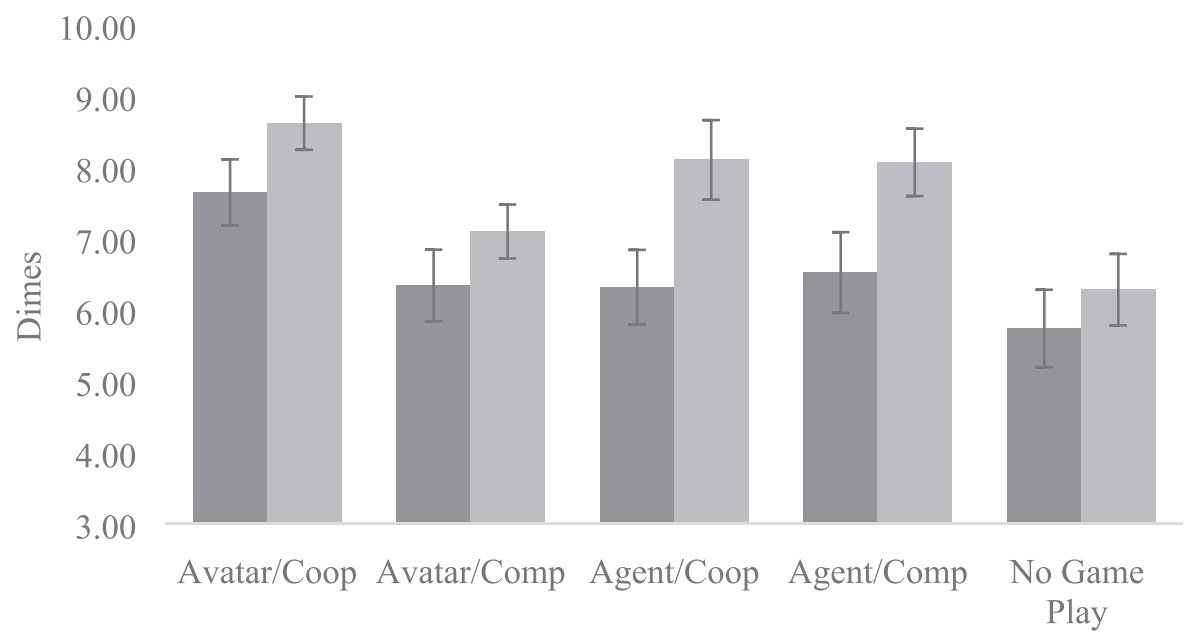

Reciprocity Expectations Donation

Figure 1 Average donation and reciprocity expectation by condition in Study 1.

to subsequent human interactions. Indeed, participants behaved more prosocially toward a stranger, relative to the control condition, even after playing competitively against an agent. ${ }^{3}$ Furthermore, the comparatively high donations to strangers after any type of game play with an agent were not mediated by increases in reciprocity expectations. Thus, the results of Experiment 1 indicated that interactions with agents did not guide participants' expectations and behaviors towards a stranger in the same way as interactions with avatars. A second experiment further examined these discrepancies and their implications for subsequent social interactions.

\section{Experiment 2}

Research has supported the predictions of BGR when avatars and humans interact consecutively (e.g., Velez, 2015) and when humans interact with the same agent consecutively (e.g., Straßmann, von der Pütten, \& Krämer, 2018). However, Experiment 1 suggested the influence of agents on BGR processes (e.g., future reciprocity expectations and prosocial behaviors towards another) might not transfer to subsequent interactants that are human. As suggested above, this may be due to a mismatch between the schemas used for agents in the first interaction and humans in the second interaction. Therefore, the second study was designed to further examine whether switching schemas in consecutive interactions with agents and humans can disrupt BGR processes.

The mindlessness of the Revised Ethopoeia Concept and the cognitive processes necessitated by consecutive social interactions may be conflicting or incompatible. While research suggests that cognitive heuristics and shortcuts lead people to apply the same social rules and expectations to interactions with avatars and agents (Sundar \& Nass, 2000), it is possible that consecutive social interactions disrupt these primarily automatic practices and encourage people to engage in more systematic processing of social information. Mindlessness has been described as "the failure to draw novel distinctions" (von der Pütten et al., 2010, p. 1642). However, perhaps when people access social information from previous interactions to inform future behaviors towards humans, the inapplicability or incongruency of schemas 
for agents becomes salient and compels people to draw a novel distinction between their previous partner (i.e., an agent) and their current human counterpart. Like trying to put a round peg in a square hole, people may be forced to acknowledge the inapplicability of prior agent interactions to subsequent behaviors towards humans.

To test this assertion in the second experiment, schema applicability was manipulated by providing participants with inapplicable or applicable targets of prosocial behaviors after playing competitively against an avatar or an agent. For example, participants who played with an agent were subsequently given an inapplicable interactant (i.e., a stranger) or an applicable interactant (i.e., an artificial intelligence agent). Participants who played competitively against an agent but subsequently interacted with a stranger would be expected to behave prosocially, similar to the first experiment, due to switching schemas across the two interactions. However, participants who subsequently interacted with an artificial intelligence agent after playing competitively against an agent would be expected to find such game play applicable, because the same schema would be used, and, therefore, the participant would behave less prosocially via lowered expectations of reciprocity. This led to the following hypotheses.

H4: Competitive game play against an agent will lead to fewer (a) reciprocity expectations and (b) prosocial behaviors towards an agent, compared to a stranger.

H5: Reciprocity expectations will mediate the reduced prosocial behaviors towards an agent, compared to a stranger, after playing competitively against an agent.

In examining the applicability of agent interactions to humans, another question that arises is the reverse relationship (i.e., the applicability of avatar interactions to agents). More specifically, do people use previous interactions with humans and avatars to guide subsequent reciprocity expectations and prosocial behaviors towards agents and artificial intelligences? As suggested by Experiment 1, agents and avatars may evoke different schemas and, thus, using human interactions to guide behaviors towards agents may result in the same urge to draw a novel distinction between interactions and, thereby, disrupt the connection. However, it is unclear whether switching from avatar interactions to agents would signal the need for active processing of social information, as argued above. For example, human schema that involve more complicated mentalizing processes, compared to agent schema, may be considered more than sufficient to guide behaviors towards agents. This may result in automatic processes akin to ethopoeia (i.e., players mindlessly use a human schema to guide behaviors towards an agent) or more active conscious processing of social information that determines the switch between schema acceptable.

RQ4: Will competitive game play against an avatar lead to different (a) reciprocity expectations and (b) prosocial behaviors towards a stranger and agent?

RQ5: Will reciprocity expectations mediate any differences in prosocial behaviors towards an agent, compared to a stranger, after playing competitively against an avatar?

\section{Methods}

\section{Participants}

Participants were 106 students ( $48.1 \%$ females; $\left.M_{\text {age }}=20.8, S D=2.82\right)$ recruited from a large Southwestern University who received course credit for their participation (see Table 1 for number of participants per condition). 


\section{Procedure}

The second study employed a 2 (agency: avatar vs agent) x 2 (subsequent interactant: human vs artificial intelligence agent) between-subjects design. The competitive conditions from Experiment 1 were recreated. After game play, participants completed post-test measures and the same modified prisoner's dilemma game from Experiment 1, but with either a stranger or an artificial intelligence agent. Participants were told the "artificial intelligence" agent in the PDG was designed to behave like a human, that it was not the "computer-controlled character you just played against," and that any money it received would go "towards its development."

\section{Results}

\section{Manipulation check}

The manipulation check was the same as in Experiment 1 for participants in the competitive conditions. A total of 13 participants failed the manipulation check and were removed from the analyses (avatars $=10$, agents $=3$ ).

\section{Analyses}

To answer the fourth hypothesis and research question, a two-way ANOVA with agency and donation partner type as fixed factors was conducted for reciprocity expectations and prosocial behaviors. Simple main effects were examined using Bonferroni adjustments. The fifth hypothesis and research question were answered using the SPSS macro PROCESS (Hayes, 2013) with 10,000 bootstrap samples. A moderated mediation model (Model 7) examined whether participants who played against an agent, compared to an avatar, behaved more or less prosocially towards an agent or human, via their expectations of reciprocity.

\section{Applicability of agent interactions to humans and artificial intelligences}

The fourth hypothesis stated that interacting with an artificial intelligence agent after playing against an agent would lead to (a) lower reciprocity expectations and (b) fewer prosocial behaviors, compared to subsequently interacting with a human. Results indicated no main or interaction effects for participants' reciprocity expectations. A simple main effect demonstrated that participants' reciprocity expectations of an artificial intelligence target $(M=4.52, S D=3.12)$ were significantly lower than their expectations of a human target $\left(M=6.37, S D=3.38 ; F[1,102]=4.8 ; p=.029 ; \eta_{\mathrm{p}}^{2}=.05\right)$ after playing against an agent. For prosocial behaviors, a marginally significant main effect for subsequent interactant type ( $F[1$, $\left.102]=3.92 ; p=.051 ; \eta_{\mathrm{p}}{ }^{2}=.04\right)$ and a significant interaction effect between agency and subsequent interactant type occurred $\left(F[1,102]=4.02 ; p=.048 ; \eta_{\mathrm{p}}^{2}=.04\right)$. A simple main effect demonstrated that participants behaved less prosocially towards an artificial intelligence target $(M=5.73, S D=3.10)$, compared to a human target $\left(M=8.04, S D=2.53 ; F[1,102]=9.13 ; p=.003 ; \eta_{\mathrm{p}}{ }^{2}=.08\right)$, after playing against an agent.

The fifth hypothesis stated that participants' reciprocity expectations would mediate their prosocial behaviors towards an artificial intelligence or human after playing against an agent. The index of moderated mediation was not significant; however, the conditional indirect effects indicated that participants' decreased prosocial behaviors towards an artificial intelligence, compared to a human, after competitive agent game play were significantly mediated by reductions in their expectations of reciprocity (point estimate: $-1.01, S E=.53$; confidence interval -2.25 to -.13 ). Therefore, the fourth and fifth hypotheses were supported. 


\section{Applicability of avatar interactions to humans and artificial intelligences}

The fourth and fifth research questions asked whether interacting with an artificial intelligence after playing against an avatar would lead to lower or higher (a) reciprocity expectations and (b) prosocial behaviors, compared to subsequently interacting with a human. Results indicated no significant differences between participants' prosocial behaviors towards an artificial intelligence agent or human after playing against an avatar. Similarly, the conditional, indirect effects of the moderated mediation analysis did not indicate that participants' reciprocity expectations mediated any behavioral differences towards an artificial intelligence agent or a human after playing against an avatar. Therefore, the fourth and fifth research questions were answered.

\section{General discussion}

The CASA (Reeves \& Nass, 1996) paradigm has mainly focused on people's initial social reactions to mediated technologies, but has overlooked how these initial, mindless reactions may influence subsequent interactions with humans. The Revised Ethopoeia Concept (Nass \& Yen, 2010) proposes that sophisticated mediated technologies, such as agents, can embody a variety of social cues and will evoke more cognitive heuristics and shortcuts as social cues are added to their repertoire. However, a growing body of research suggests that social cues may not be enough to activate mentalizing with agents (see Fox et al., 2015). This lack of mentalizing, potentially due to applying a nonhuman schema (Merritt, 2012), may limit the range of social reactions people can have towards agents (e.g., de Melo et al., 2014). That is, the more a social interaction relies on mentalizing, the more reactions to agents and humans (e.g., avatars) will diverge.

The current two experiments demonstrate that applying different schema to agents and avatars has implications beyond initial encounters for subsequent interactions. Experiment 1 suggests that interactions with agents are not used to guide reciprocity expectations and prosocial behaviors towards a stranger, unlike avatar interactions, which informed participants' subsequent social decisions, based on the cooperative or competitive nature of game play. It was proposed that the mismatch between a nonhuman schema used for agents during game play and a human schema used for strangers in the subsequent interaction might have led participants to engage in more systematic processing of relevant social information and, thus, made the incongruency between schema more salient. If consecutive interactions forced participants to acknowledge the inapplicability of the agent schema to humans, then perhaps it was easier for people to become mindful of agents' nonhuman status than was suggested by the Revised Ethopoeia Concept.

The second experiment corroborated the assertion that incongruent schema between game play with agents and subsequent interactions with humans can disrupt the predictions of BGR (Yamagishi et al., 1999), a theory commonly used for consecutive social interactions. For example, it was demonstrated that allowing participants to interact with an artificial intelligence agent after competing against an agent aligned the schema used during consecutive social interactions and, thus, permitted game play to guide participants' subsequent social decisions. However, the second study also demonstrated that avatar interactions can guide people's reciprocity expectations and prosocial behaviors towards a subsequent artificial intelligence agent (see Figure 2). This suggests that human schema are perceived as applicable to both avatars and agents, while agent schema may only be applied to agents (e.g., artificial intelligences). This may be due to human schema subsuming agents and, thereby, avoiding any perceived (conscious or unconscious) conflict during consecutive social interactions with humans and then artificial intelligences. 


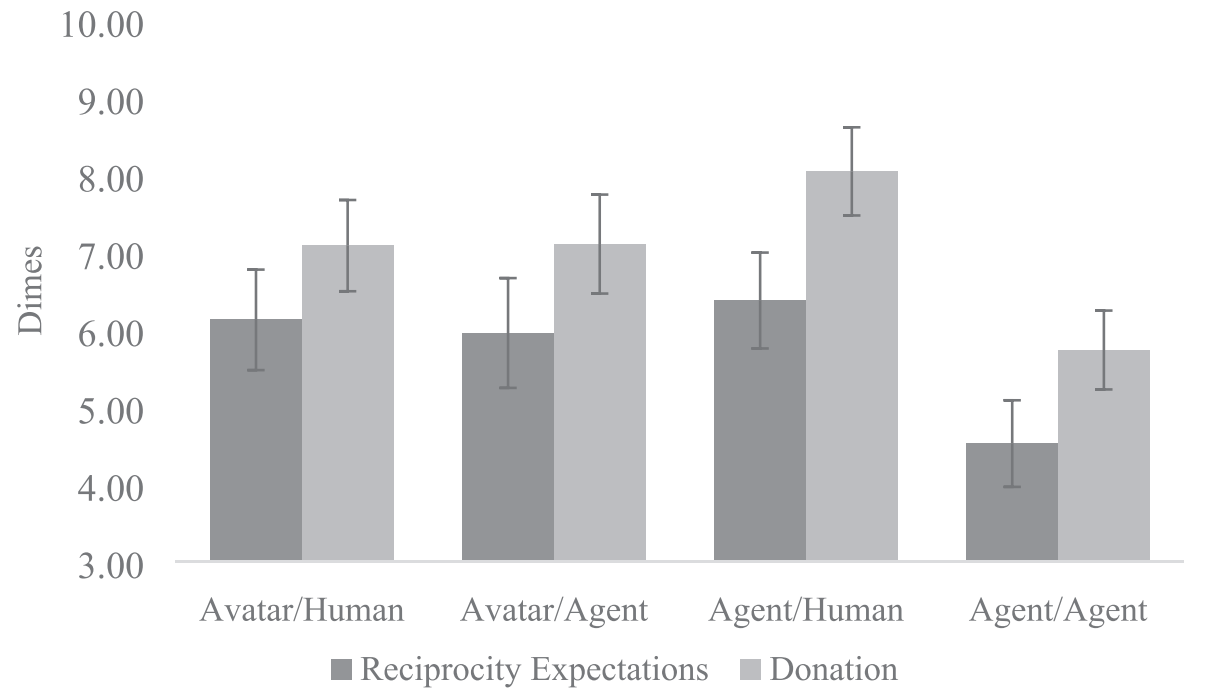

Figure 2 Average donation and reciprocity expectation by condition in Study 2.

The results also suggest that switching schemas has different implications based on the order of schema activated and the target of prosocial behaviors. For example, participants in the first study were more prosocial towards a stranger after an interaction with an agent, regardless of the game play type, compared to those in the control condition (i.e., no game play at all). ${ }^{4}$ Perhaps the inability to use a recent interaction to inform social decisions made participants more uncertain, causing them to err on the side of caution as compared to participants who did not have conflicting schemas (e.g., the control condition). Indeed, research has suggested that people are naturally concerned with being fair in social interactions (see Fehr \& Schmidt, 1999), and the systematic processing of social information resulting from inconsistent schema may have heightened this concern. Research has suggested that people do not have this concern when interacting with agents (de Melo et al., 2016), potentially due to the lack of mentalizing. Therefore, in the second study, it is possible that people's substantially lower donations to an artificial intelligence agent after playing against an agent resulted from the lack of guilt people feel after cheating agents.

Future research should further examine the composition of agent schemas and monitor their evolution as agent technology advances and becomes a familiar presence. For example, in today's world, people do not want to ascribe the capacity to sense and feel (i.e., experience) to agents, because agents are perceived as lacking a mind capable of these processes (Gray, Gray, \& Wegner, 2007). Indeed, people feel uneasy when prompted to ascribe this dimension of mind to an agent (Gray \& Wegner, 2012). However, if it becomes commonplace to perceive future agents as capable of experiencing feelings and sensations, then people may ascribe more mind to agents and, thus, not need to switch schemas for subsequent human interactions. Research already suggests that people are willing to assign more human traits to agents as they become more human-like (e.g., Bergmann et al., 2015; Krach et al., 2008). Research should identify which characteristics to bestow upon agents, such as the ability to experience feelings and sensations, that reduce the need for schema switching in consecutive interactions with agents and humans. 


\section{Notes:}

1. Outliers were assessed using boxplots for each condition in SPSS after removing participants who had failed the manipulation check. Results indicated that prosocial behaviors of four participants in the cooperative avatar condition were at least 1.5 box lengths from the lower hinge of the box. Of these participants, three gave zero dimes (2.64 SDs below mean), while the other gave a single dime (2.35 SDs below mean). The mean for prosocial behaviors in the cooperative avatar condition became 8.78, with a standard deviation of 3.32. The analyses for H1b, H2a, H3b, and RQ3 were nonsignificant when including the outliers, while $\mathrm{H} 2 \mathrm{~b}(p=.052 ; \mathrm{d}=.46)$ and RQ2 became marginally significant $\left(p=.088 ; \eta_{\mathrm{p}}{ }^{2}=.02\right)$

2. A significant Chi-square analysis indicated that participants in the avatar conditions failed the manipulation check significantly more often than participants in the agent conditions $(\chi[1]=5.33$; $p=.02$ ). More data were collected in the avatar conditions to compensate. The response options of the manipulation check question that included the possibility of deception, in conjunction with the more complicated deception for the avatar conditions, may have unduly instilled suspicion in the manipulation.

3. The reported contrasts were not orthogonal, but Bonferroni adjustments to their $p$ values allowed a more conservative interpretation of their results.

4. A $t$-test (equal variances not assumed) indicated that playing competitively against an agent led to more prosocial behaviors towards a stranger $(M=8.06, S D=2.72)$, compared to not playing at all (i.e., the control condition, $M=6.28, S D=3.17 ; t[70.88]=2.59 ; p=.012$ ).

\section{References}

Appel, J., von der Pütten, A., Krämer, N., \& Gratch, J. (2012). Does humanity matter? Analyzing the importance of social cues and perceived agency of a computer system for the emergence of social reactions during human-computer interaction. Advances in Human-Computer Interaction, 2012: 1-10. doi: 10.1155/2012/324694.

Bergmann, K., Branigan, H. P., \& Kopp, S. (2015). Exploring the alignment space-lexical and gestural alignment with real and virtual humans. Frontiers in ICT, 36: 1-11. doi: 10.3389/fict.2015.00007.

Bettenhausen, K. L., \& Murnighan, J. K. (1991). The development of an intragroup norm and the effects of interpersonal and structural challenges. Administrative Science Quarterly, 36: 20-35. doi: $10.2307 / 2393428$.

Ewoldsen, D. R., Eno, C. A., Okdie, B. M., Velez, J. A., Guadagno, R. E., \& DeCoster, J. (2012). Effect of playing violent video games cooperatively or competitively on subsequent cooperative behavior. Cyberpsychology, Behavior, and Social Networking, 15(5), 277-280. doi: 10.1089/cyber.2011.0308.

Fehr, E., \& Schmidt, K. (1999). A theory of fairness, competition, and cooperation. Quarterly Journal of Economics, 114, 817-868. doi: 10.1162/003355399556151.

Fox, J., Ahn, S. J., Janssen, J. H., Yeykelis, L., Segovia, K. Y., \& Bailenson, J. N. (2015). Avatars versus agents: A meta-analysis quantifying the effect of agency on social influence. Human-Computer Interaction, 30(5), 401 432. doi: 10.1080/07370024.2014.921494.

Gallagher, H. L., Jack, A. I., Roepstorff, A., \& Frith, C. D. (2002). Imaging the intentional stance in a competitive game. Neuroimage, 16(3), 814-821. doi: 10.1006/nimg.2002.1117.

Greitemeyer, T., \& Cox, C. (2013). There's no "I" in team: Effects of cooperative video games on cooperative behavior. European Journal of Social Psychology, 43(3), 224-228. doi: 10.1002/ejsp.1940. 
Greitemeyer, T., Traut-Mattausch, E., \& Osswald, S. (2012). How to ameliorate negative effects of violent video games on cooperation: Play it cooperatively in a team. Computers in Human Behavior, 28(4), 1465-1470. doi: 10.1016/j.chb.2012.03.009.

Gray, H. M., Gray, K., \& Wegner, D. M. (2007). Dimensions of mind perception. Science, 315(5812), 619-619. doi: 10.1126/science.1134475.

Gray, K., \& Wegner, D. M. (2012). Feeling robots and human zombies: Mind perception and the uncanny valley. Cognition, 125(1). doi: 10.1126/science.1134475.

Guadagno, R. E., Blascovich, J., Bailenson, J. N., \& McCall, C. (2007). Virtual humans and persuasion: The effects of agency and behavioral realism. Media Psychology, 10, 1-22 doi: $10.1080 / 15213260701300865$.

Hayes, A. F. (2013). Introduction to Mediation, Moderation, and Conditional Process Analysis A Regression-Based Approach, New York, NY: The Guilford Press.

Kircher, T., Blümel, I., Marjoram, D., Lataster, T., Krabbendam, L., Weber, J., .. \& Krach, S. (2009). Online mentalising investigated with functional MRI. Neuroscience letters, 454(3). 176-181 doi: 10.1016/j.neulet.2009.03.026.

Krach, S., Hegel, F., Wrede, B., Sagerer, G., Binkofski, F., \& Kircher, T. (2008). Can machines think? Interaction and perspective taking with robots investigated via fMRI. PLOS One, 3(7), e2597. doi: 10.1371/journal.pone.0002597.

Krämer, N. C., Eimler, S., von der Pütten, A. \& Payr, S. (2011) . Theory of companions: what can theoretical models contribute to applications and understanding of human-robot interaction?, Applied Artificial Intelligence, 26: 474-502. doi: 10.1080/08839514.2011.587153.

Krämer, N. C., Rosenthal-von der Pütten, A. M., \& Hoffmann, L. (2015). Social effects of virtual and robot companions. In S. S. Sundar (Ed.) The handbook of the psychology of communication technology, p. 137-159, Malden, MA: John Wiley \& Sons, Inc.

Lang, A., \& Ewoldsen, D. (2010). Beyond effects. In In S. Allan (Ed.), Rethinking communication (pp. 111-122). Cresskill, NJ: Hampton Press.

Lim, S., \& Reeves, B. (2010). Computer agents versus avatars: Responses to interactive game characters controlled by a computer or other player. International Journal of Human-Computer Studies, 68(12), 57-68. doi: 10.1016/j.ijhcs.2009.09.008.

de Melo, C. M., Carnevale, P. J., \& Gratch, J. (2014). Using virtual confederates to research intergroup bias and conflict. In Academy of Management Proceedings (Vol. 2014, No. 1, p. 11226). Briarcliff Manor, NY: Academy of Management.

de Melo, C. M., Gratch, J., \& Carnevale, P. J. (2013, September). The effect of agency on the impact of emotion expressions on people's decision making. In Guclu, ATinc In 2013 Humaine Association Conference on Affective Computing and Intelligent Interaction (pp. 546-551). Danvers, MA: IEEE.

de Melo, C., Marsella, S., \& Gratch, J. (2016). People do not feel guilty about exploiting machines. In Juan, E. Guerrero, ACM Transactions on Computer-Human Interaction (TOCHI), 23(2), 1-17. doi:10.1145/2890495

Merritt, T. R. (2012). A failure of imagination: How and why people respond differently to human and computer team-mates. Doctoral dissertation National University of Singapore.

Nass, C., Fogg, B. J., \& Moon, Y. (1996). Can computers be teammates? International Journal of HumanComputer Studies, 45(6), 669-678. doi: 10.1006/ijhc.1996.0073.

Nass, C., \& Moon, Y. (2000). Machines and mindlessness: Social responses to computers. Journal of Social Issues, 56(1), 81-103. doi: 10.1111/0022-4537.00153.

Nass, C., \& Yen, C. (2010). The man who lied to his laptop: What we can learn about ourselves from our machines. New York, NY: Penguin. 
Nowak, K. L., \& Fox, J. (2018). Avatars and computer-mediated communication: A review of the definitions, uses, and effects of digital representations. Review of Communication Research, 6, 30-53. doi: 10.12840/issn.2255-4165.2018.06.01.015.

Parise, S., Kiesler, S., Sproull, L., \& Waters, K. (1999). Cooperating with life-like interface agents. Computers in Human Behavior, 15(2), 123-142. doi: 10.1016/S0747-5632(98)00035-1.

von der Pütten, A., Krämer, N., Gratch, J., \& Kang, S.-H. (2010). “It doesn't matter what you are!” Explaining social effects of agents and avatars. Computers in Human Behavior, 26(6), 1641-1650. doi: $10.1016 /$ j.chb.2010.06.012.

von der Pütten, A., Schulte, F. P., Eimler, S. C., Sobieraj, S., Hoffmann, L., Maderwald, S., . . \& Krämer, N. C. (2014). Investigations on empathy towards humans and robots using fMRI. Computers in Human Behavior, 33, 201-212. doi: 10.1016/j.chb.2014.01.004

Reeder, G. D., Kumar, S., Hesson-McInnis, M. S., \& Trafimow, D. (2002). Inferences about the morality of an aggressor: The role of perceived motive. Journal of Personality and Social Psychology, 83(4), 789-803. doi: 10.1037/0022-3514.83.4.789

Reeves, B., \& Nass, C. I. (1996). The media equation: How people treat computers, television, and new media like real people and places. New York, NY: Cambridge University Press.

Rothmund, T., Gollwitzer, M., \& Klimmt, C. (2011). Of virtual victims and victimized virtues: Differential effects of experienced aggression in video games on social cooperation. Personality and Social Psychology Bulletin, 37(1), 107-119. doi: 10.1177/0146167210391103.

Schmierbach, M. (2010). "Killing spree": Exploring the connection between competitive game play and aggressive cognition. Communication Research, 37(2), 256-274. doi: 10.1177/0093650209356394.

Straßmann, C., von der Pütten, A., \& Krämer, N. (2018). With or against each other? The influence of a virtual agent's (non) cooperative behavior on user's cooperation behavior in the prisoners' dilemma. Advances in Human-Computer Interaction, 2018, 1-7. doi: 10.1155/2018/2589542.

Sundar, S., \& Nass, C. (2000). Source orientation in human-computer interaction: Programmer, networker, or independent social actor. Communication Research, 27(6), 683-703. doi: 10.1177/009365000027006001

Tajfel, H., Bilig, M. G., Bundy, R. P., \& Flament, C. (1971). Social categorization and intergroup behavior. European Journal of Social Psychology, 1, 149-178. doi: 10.1002/ejsp.2420010202.

Van Lange, P. A., \& Kuhlman, D. M. (1994). Social value orientations and impressions of partner's honesty and intelligence: A test of the might versus morality effect. Journal of Personality and Social Psychology, 67(1), 126-141. doi: 10.1037/0022-3514.67.1.126.

Velez, J. A. (2015). Extending the theory of bounded generalized reciprocity: An explanation of the social benefits of cooperative video game play. Computers in Human Behavior, 48, 481-491. doi: $10.1016 /$ j.chb.2015.02.015.

Velez, J. A. (2018). Incubators of prosocial behaviors: An evolutionary approach to understanding dynamic social video game interactions. In J. Breuer, D. Pietschmann, B. Liebold \& B. P. Lange (Eds.), Evolutionary psychology and digital games: Digital hunter-gatherers. 134-144, New York, NY: Routledge.

Velez, J. A., Greitemeyer, T., Whitaker, J. L., Ewoldsen, D. R., \& Bushman, B. J. (2016). Violent video games and reciprocity: The attenuating effects of cooperative game play on subsequent aggression. Communication Research, 43(4), 447-467. doi: 10.1177/0093650214552519.

Velez, J. A., Mahood, C., Ewoldsen, D. R., \& Moyer-Gusé, E. (2014). Ingroup versus outgroup conflict in the context of violent video game play: The effect of cooperation on increased helping and decreased aggression. Communication Research, 41(5), 607-626. doi: 10.1177/0093650212456202. 
Waddell, J. C., \& Peng, W. (2014). Does it matter with whom you slay? The effects of competition, cooperation and relationship type among video game players. Computers in Human Behavior, 38, 331-338. doi: 10.1016/j.chb.2014.06.017.

Waytz, A., Gray, K., Epley, N., \& Wegner, D. M. (2010). Causes and consequences of mind perception. Trends in Cognitive Sciences, 14(8), 383-388. doi: 10.1016/j.tics.2010.05.006.

Weibel, D., Wissmath, B., Habegger, S., Steiner, Y., \& Groner, R. (2008). Playing online games against computer-vs. human-controlled opponents: Effects on presence, flow, and enjoyment. Computers in Human Behavior, 24(5), 2274-2291. doi: 10.1016/j.chb.2007.11.002.

Yamagishi, T., Jin, N., \& Kiyonari, T. (1999). Bounded generalized reciprocity: Ingroup boasting and ingroup favoritism. Advances in Group Processes, 16(1), 161-197. 